\title{
GERENCIAMENTO DE RESÍDUOS QUÍMICOS EM UM LABORATÓRIO DE ENSINO E PESQUISA: A EXPERIÊNCIA DO LABORATÓRIO DE LIMNOLOGIA DA UFRJ
}

\author{
Claudio Cardoso Marinho*, Reinaldo Luiz Bozelli e Francisco de Assis Esteves \\ Laboratório de Limnologia, Departamento de Ecologia, Instituto de Biologia, Universidade \\ Federal do Rio de Janeiro
}

Av. Rodolpho Paulo Rocco, s/n. Centro de Ciências da Saúde, Bloco A, sala A-08

CEP 21.941-902, Rio de Janeiro, RJ.

Ana Claudia Braga Gonçalves, Vanessa de Almeida Rocha e Wesley Higino da Silva Instituto Federal de Educação, Ciência e Tecnologia do Rio de Janeiro

Rua Lúcio Tavares, 1045. 26530-060, Nilópolis, RJ Julio Carlos Afonso

Departamento de Química Analítica, Instituto de Química da Universidade Federal do Rio de Janeiro

Av. Athos da Silveira Ramos, 149 Bloco A, sala A-509. 21941-909 Rio de Janeiro, RJ

*clcamar@biologia.ufr.br 
CHEMICAL WASTE MANAGEMENT IN A TEACHING AND RESEARCH LABORATORY: THE EXPERIENCE OF THE LABORATORY OF LIMNOLOGY OF UFRJ

This work describes the waste management system developed at the Laboratory of Limnology of Federal University of Rio de Janeiro. The initial challenge was to identify and treat a great variety of wastes generated in the analytical procedures performed in the laboratory. Since many of the students and researchers are not chemists, an intensive training was performed during the establishment of the management system. After three years, there was a deep change of attitude of the professionals involved. The present experience may be useful for other laboratories with a profile similar to the one under study.

Keywords: chemical wastes; waste management; laboratory wastes

\section{Introdução}

Os resíduos químicos de laboratório gerados por atividades de pesquisa e/ou ensino nas universidades e centros de pesquisa passaram a ser uma preocupação no Brasil a partir da década de 1990 face a até então falta de um gerenciamento adequado para os mesmos. Essa questão não se restringe apenas à adoção de práticas que visem à minimização e ao tratamento dos resíduos produzidos nas atividades laboratoriais, mas também a conscientização e treinamento do fator humano [1,2], já que não basta apenas dispor de rotas de tratamento de resíduos se as pessoas não são parte ativa e integrante da gestão dos mesmos. Com o aumento e a diversificação das discussões relativas a problemas e impactos ambientais decorrentes das atividades humanas tornou-se intolerável a falta de compromisso com a gestão dos resíduos químicos gerados nas instituições de ensino e pesquisa, locais por excelência de formação de novos recursos humanos [3]. Outra característica dessas instituições é que elas não eram habitualmente consideradas unidades poluidoras. Culturalmente sempre se buscou apenas focar o ensino e a instrução técnica dos alunos. Por isso, durante décadas, muitos dos procedimentos nessas instituições geraram quantidades desnecessárias de resíduos por empregarem grandes quantidades de reagentes, e que 
eram descartados sem critério algum [4]. Isso reflete bem o modelo que vigorou por muito tempo, o do desenvolvimento sem qualquer noção de sustentabilidade ambiental $[5,6]$.

A literatura mostra diversos trabalhos envolvendo resíduos químicos de laboratório, os quais se enquadram em dois tipos básicos: o estabelecimento de rotas de gestão, focando o tratamento de correntes residuais para um descarte final adequado e/ou a recuperação de elementos de elevado valor agregado [2,3,7-13], e a avaliação do impacto desses programas de gestão sobre as pessoas envolvidas nos mesmos, com ênfase na percepção e assimilação de uma nova cultura comprometida com o meio ambiente e a gestão dos resíduos gerados por eles mesmos $[3,7,9,10,14-16]$.

A grande parte dessas experiências se ambienta em laboratórios de ensino e pesquisa em química. Parecem existir poucos relatos em laboratórios de áreas correlatas como farmácia, biologia e nutrição [17]. Naturalmente, nestes laboratórios, também existe toda uma variedade de análises químicas. Da mesma forma, as pessoas que trabalham nesses locais precisam conscientizar-se e ser formadas para gerir de forma efetiva os resíduos. O estabelecimento desses programas de gestão é uma excelente oportunidade de aprendizagem, formação e sensibilização para alunos, professores e técnicos. O fator humano deve ser valorizado, na medida em que todos os usuários são parte integrante do programa e corresponsáveis pelos resultados e avaliações a serem obtidos [1].

O tratamento de um resíduo aquoso de laboratório exige a aplicação dos conhecimentos básicos de equilíbrio químico [18]: neutralização ácido-base; escolha do agente precipitante de metais pesados e/ou de ânions presentes no resíduo, minimizando riscos de solubilização dos mesmos por complexação; aplicação de reações de oxirredução para oxidar/reduzir espécies presentes no resíduo. O efluente final deve ser límpido e incolor, ter $\mathrm{pH}$ em torno de 7 e apresentar caráter redox indiferente $[8,9,19]$.

O laboratório de Limnologia da Universidade Federal do Rio de Janeiro (UFRJ), criado em 1989, caracteriza-se pela realização de várias atividades de pesquisa em virtude do grande número de alunos de doutorado e de mestrado, de programas de estágio para alunos de iniciação científica, e de projetos junto a agências de fomento e à iniciativa privada. Além disso, outra atividade geradora de resíduos é as aulas práticas de graduação para os cursos de bacharelado em 
Ecologia do Instituto de Biologia da UFRJ e do Programa de Pós Graduação em Ecologia da mesma universidade.

O tratamento dos resíduos gerados em atividades de pesquisa pode, a princípio, ser difícil em virtude da complexidade do universo de trabalho [11.20]. No caso do laboratório de Limnologia, as características das amostras podem variar bastante em função do local de coleta, do tipo de amostra e da natureza da análise. Variáveis como $\mathrm{pH}$, salinidade, potencial redox e a quantidade ou qualidade da matéria orgânica (MO) variam enormemente em função da condição do ambiente [21]. Além da análise de água, o laboratório realiza também análises de sedimento e de plantas aquáticas, aumentando ainda mais a diversidade de análises realizadas.

A implementação de um programa de gerenciamento de resíduos passa por uma tomada de consciência acerca da necessidade de adotar novos hábitos no sentido de atender não só a legislação vigente, mas principalmente a uma nova mentalidade que se preocupe não apenas com a qualidade das análises, mas também com a gestão dos resíduos. Essa visão passa pela identificação, tratamento e encaminhamento dos mesmos, de forma a diminuir os possíveis impactos ao meio ambiente.

Com o objetivo de servir como uma experiência-modelo para laboratórios congêneres, este trabalho descreve a implementação de um programa de gestão de resíduos no Laboratório de Limnologia da UFRJ. A primeira fase foi o mapeamento dos diversos procedimentos geradores de resíduos, primordial para a identificação das diversas correntes residuais. A partir dessa identificação adotaram-se estratégias de tratamento de cada corrente com vistas a eliminar as características perigosas (corrosividade, metais pesados em solução, reagentes orgânicos de elevada toxicidade e etc.) ou então definir uma destinação final adequada (incineração, coprocessamento etc.). Ao longo de todo esse processo, as pessoas envolvidas receberam treinamento adequado. Este trabalho também aborda a receptividade dessas pessoas frente à implementação do programa de gestão.

\section{Materiais e métodos}




\subsection{Origem das correntes residuais do laboratório}

Todo processo de implementação de um sistema de gestão de resíduos exige como prérequisito a identificação dos procedimentos laboratoriais que geram as diversas correntes residuais. Isso se reveste de capital importância porque a identificação dessas correntes previne alguns problemas que podem ocorrer na estocagem (como a reação entre componentes incompatíveis oriundos de correntes misturadas), bem como permite muitas vezes planejar a mistura de determinadas correntes de modo a eliminar muitas de suas características tóxicas (por exemplo, a mistura de uma corrente ácida com uma alcalina evita o uso de reagentes para neutralização individual e permite a precipitação direta de metais pesados). Via de regra os resíduos químicos são classificados como perigosos (Classe I) segundo a Norma NBR 10004 [22] porque quase sempre apresentam pelo menos uma das seguintes características: reatividade, inflamabilidade, corrosividade e toxicidade. A Norma NBR 12235 (armazenamento de resíduos perigosos) explicita as regras a serem seguidas para evitar situações de incompatibilidade química [23]. O manejo de resíduos é um exemplo de atividade insalubre, de acordo com a NR15 do Ministério do Trabalho [24], exigindo para tal o emprego de equipamentos de proteção individual (EPIs) adequados.

Uma vez explicado ao pessoal do laboratório as regras básicas, foi feito o processo de identificação dos procedimentos geradores de resíduos. Esses últimos correspondem à análise de águas, sedimentos ou plantas aquáticas de vários ecossistemas aquáticos brasileiros (como lagoas costeiras, rios e lagos amazônicos). As análises químicas nas amostras de água são: (a) fósforo total, fósforo dissolvido e ortofosfato, através da reação de formação do complexo com ácido fosfomolíbdico [25]; (b) nitrogênio amoniacal pelo método de Kejdahl [26]; (c) clorofila, realizada através de extração com etanol $90 \%$ a $80{ }^{\circ} \mathrm{C}$ em material particulado [25]; (d) oxigênio dissolvido na água (OD) segundo método de Winkler, modificado por Golterman [25]; (e) fixação de amostras com $\mathrm{ZnCl}_{2}$, com o objetivo de inibir qualquer atividade biológica [27].

Em relação ao sedimento e às plantas aquáticas utilizam-se os seguintes métodos: (a) fósforo total, realizado através de digestão obtida através de mistura de ácidos a $300{ }^{\circ} \mathrm{C}$, e posterior reação através da formação de complexo azul de fosfomolíbdico [28]; (b) nitrogênio amoniacal (Kejdahl), através da digestão a $350{ }^{\circ} \mathrm{C}$ com ácido sulfúrico em presença de catalisador à base de $\mathrm{Cu}$ e Se [29]. 
A composição exata das correntes residuais não pode ser totalmente conhecida, em função da diversidade das amostras ambientais que são analisadas por cada metodologia. Contudo, a Tabela 1 fornece as principais características de cada corrente identificada, enquanto que a Tabela 2 lista os ânions e cátions presentes em cada resíduo. Todas as informações se baseiam nas quantidades dos reagentes adicionadas para cada ensaio acima descrito. A Figura 1 mostra um exemplo de um fluxograma descritivo de como cada corrente residual é gerada. A Figura 2 mostra uma atitude muito importante na fase inicial de um programa de gestão: a identificação das diversas correntes residuais com as informações básicas necessárias ao seu processamento.

Tabela 1: Dados de caracterização visual e físico-química dos resíduos iniciais

\begin{tabular}{|c|c|c|c|c|}
\hline $\begin{array}{c}\text { Elemento ou } \\
\text { composto de interesse }\end{array}$ & Descrição & pH & $\begin{array}{c}\text { Volume tratado } \\
(\mathrm{mL})\end{array}$ & $\begin{array}{c}\text { Caráter } \\
\text { redox }\end{array}$ \\
\hline Mo e $\mathrm{Sb}$ & $\begin{array}{l}\text { Solução azul sem } \\
\text { precipitado }\end{array}$ & 2 & 15000 & Redutor \\
\hline $\mathrm{Hg}$ & $\begin{array}{l}\text { Solução incolor + } \\
\text { precipitado escuro }\end{array}$ & 0 & 10000 & Redutor \\
\hline Etanol & $\begin{array}{l}\text { Solução verde sem } \\
\text { precipitado }\end{array}$ & 2 & 2000 & Redutor \\
\hline $\mathrm{Zn}$ & $\begin{array}{l}\text { Solução incolor + } \\
\text { precipitado claro }\end{array}$ & 6 & 5000 & Indiferente \\
\hline $\mathrm{Cu}$ e Se & $\begin{array}{l}\text { Solução incolor + } \\
\text { precipitado escuro }\end{array}$ & $8-9$ & 10000 & Redutor \\
\hline Mn e I & $\begin{array}{l}\text { Solução amarela } \\
\text { sem precipitado }\end{array}$ & 2 & 5000 & Redutor \\
\hline
\end{tabular}


Tabela 2: Lista de espécies químicas presentes nas correntes de resíduos identificadas

\begin{tabular}{|c|c|c|c|}
\hline $\begin{array}{l}\text { Elemento } \\
\text { composto } \\
\text { interesse }\end{array}$ & ${ }_{\text {de }}^{\text {ou }}$ Outros cátions & Ânions & Outros compostos \\
\hline Mo e Sb & $\mathrm{H}^{+}$ & $\mathrm{PO}_{4}{ }^{3-}, \mathrm{SO}_{4}{ }^{2-}$ & $\begin{array}{c}\text { ácido ascórbico, íon } \\
\text { tartarato e } \mathrm{MO}^{*}\end{array}$ \\
\hline Hg & $\mathrm{Na}^{+}, \mathrm{K}^{+}, \mathrm{NH}_{4}^{+}$ & $\mathrm{SO}_{4}^{2-}$ & MO \\
\hline Etanol & $\mathrm{H}^{+}$ & $\mathrm{Cl}^{-}$ & MO e pigmentos \\
\hline Zn & & $\mathrm{Cl}^{-}$ & MO \\
\hline Cu e Se & $\mathrm{Na}^{+}, \mathrm{K}^{+}$ & $\mathrm{Cl}^{-}, \mathrm{SO}_{4}^{2-}, \mathrm{OH}^{-}$ & MO \\
\hline Mn e I & $\mathrm{Na}^{+}, \mathrm{K}^{+}$ & $\begin{array}{c}\mathrm{SO}_{4}{ }^{2-}, \mathrm{SO}_{3}{ }^{2-}, \mathrm{OH}^{-} \\
\mathrm{S}_{4} \mathrm{O}_{6}{ }^{2-}\end{array}$ & MO \\
\hline
\end{tabular}

* MO: matéria orgânica 


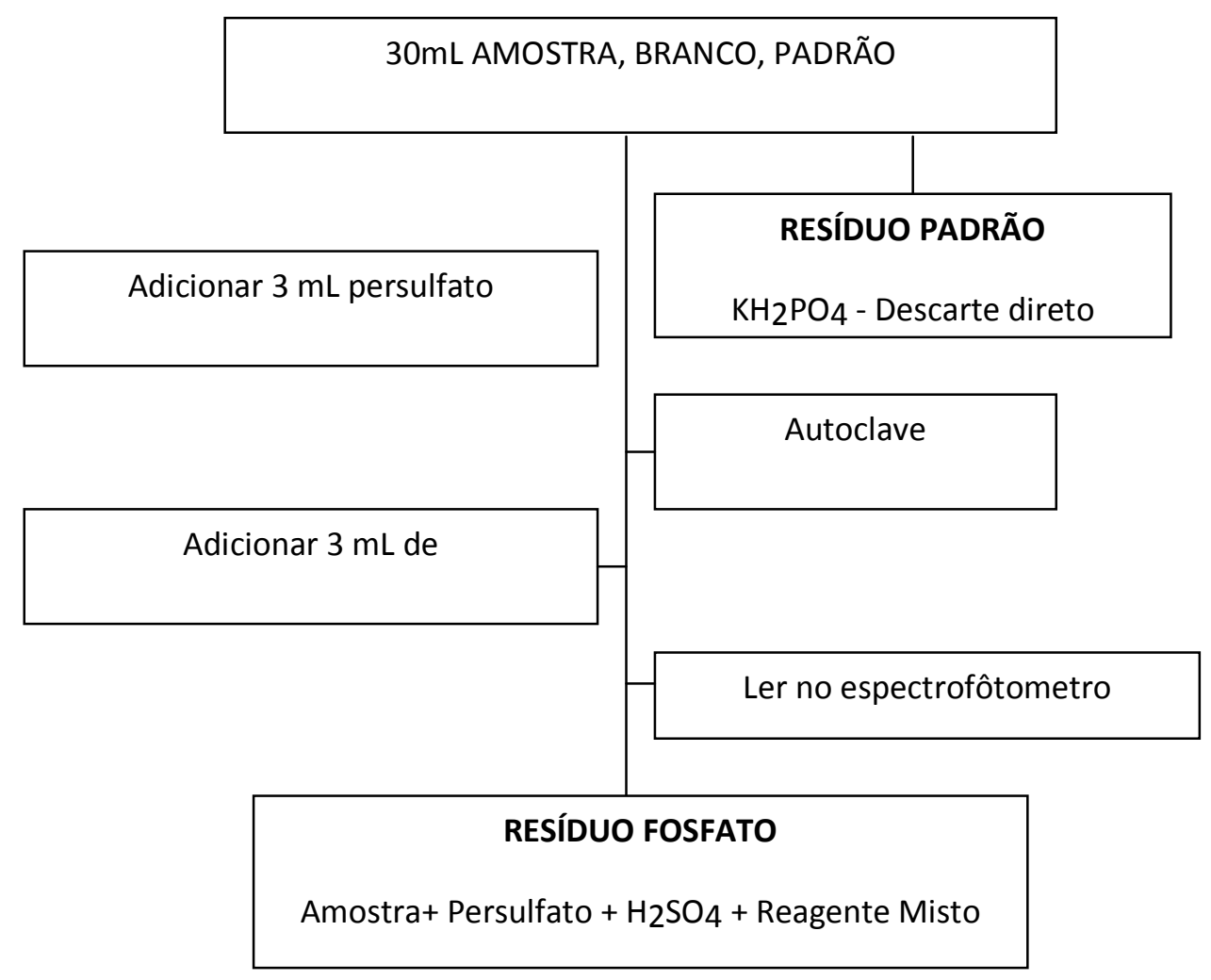

Figura 1: Exemplo de fluxograma utilizado em cada análise, exemplificado para o caso da determinação de fosfato, com objetivo de identificar os rejeitos produzidos no procedimento 


\section{Laboratório de Limnologia - Resíduos}

Análise: Fosfato Dissolvido

Nome: Resíduo Fosfato

Composição: Amostra/Branco/Padrão, $\mathrm{H}_{2} \mathrm{SO}_{4}$, Persulfato, Reagente

Misto

Características: resíduo redutor, pH ácido, metais pesados solúveis

Observações:

Figura 2: Exemplo de rótulo para identificação das correntes de resíduos

\subsection{Seleção das rotas de tratamento de cada corrente residual identificada}

Análise de clorofila. O tratamento consiste na destilação do resíduo para o isolamento do etanol. O material não destilado, de consistência aquosa, foi neutralizado com $\mathrm{NaOH}$ 12,5 mol.L $\mathrm{L}^{-1}$ até pH 7-8; o precipitado, marrom, decantou rapidamente. O sobrenadante foi retirado através de um sifão, e a massa pastosa foi filtrada em um papel de filtro. O precipitado foi colocado em recipiente plástico. O sobrenadante era esverdeado.

Análise de fósforo. A neutralização foi o método escolhido, o que permite não só eliminar a corrosividade do resíduo, mas também separar por precipitação os metais pesados presentes no mesmo. Para cada $1 \mathrm{~L}$ de resíduo adicionou-se cerca de $30 \mathrm{ml}$ de $\mathrm{NaOH} 50 \%$ (12,5 mol. $\left.\mathrm{L}^{-1}\right)$ até pH 8, para que o antimônio precipitasse na forma de sais do íon antimonila $\left(\mathrm{SbO}^{+}\right)$. A adição de $350 \mathrm{ml}$ de $\mathrm{BaCl}_{2} 2$ mol. $\mathrm{L}^{-1}$ completou a precipitação dos íons $\mathrm{SbO}^{+}, \mathrm{MoO}_{4}{ }^{2-}$ (molibdato) e do fósforo. Caso haja coloração azul (azul de molibdênio), deve-se adicionar um pouco de $\mathrm{H}_{2} \mathrm{O}_{2}$ a $3 \%$ a fim de oxidar todo o molibdênio a molibdato. A decantação total do sólido (branco) produzido ocorreu em $24 \mathrm{~h}$. O sobrenadante foi tratado com solução de $\mathrm{Na}_{2} \mathrm{SO}_{4}$ 0,5 mol.L $\mathrm{L}^{-1}$ para 
remoção do excesso de $\mathrm{Ba}^{+2}$. Os precipitados foram armazenados em local à parte para a destinação final. As reações que ocorrem nos procedimentos acima são as seguintes:

$$
\begin{aligned}
& \mathrm{SbO}^{+}+\mathrm{OH}^{-} \rightarrow \mathrm{SbOOH} \downarrow \\
& 2 \mathrm{SbO}^{+}+\mathrm{BaCl}_{2} \rightarrow 2 \mathrm{SbOCl} \downarrow+\mathrm{Ba}^{+2} \\
& \mathrm{MoO}_{4}{ }^{2-}+\mathrm{BaCl}_{2} \rightarrow \mathrm{BaMoO}_{4} \downarrow+2 \mathrm{Cl}^{-} \\
& 2 \mathrm{PO}_{4}{ }^{3-}+3 \mathrm{BaCl}_{2} \rightarrow \mathrm{Ba}_{3}\left(\mathrm{PO}_{4}\right)_{2} \downarrow+6 \mathrm{Cl}^{-} \\
& \mathrm{SO}_{4}{ }^{2-}+\mathrm{BaCl}_{2} \rightarrow \mathrm{BaSO}_{4} \downarrow+2 \mathrm{Cl}^{-}
\end{aligned}
$$

Análise de nitrogênio em água. Este resíduo tem características que o diferenciam dos demais por conta da presença do mercúrio. A neutralização da solução ácida com $\mathrm{NaOH}$ 12,5 mol.L $\mathrm{L}^{-1}$ até pH 8 precipitou o mercúrio como $\mathrm{Hg}(\mathrm{OH})_{2}$, que foi filtrado em papel de filtro. O sobrenadante e o precipitado foram encaminhados para a destinação final.

Análise de nitrogênio em sedimento e macrófita. Este resíduo foi tratado com solução de $\mathrm{NaOH}$ 12,5 mol. $\mathrm{L}^{-1}$ até $\mathrm{pH}$ entre 10 e 12, onde o cobre precipita como $\mathrm{Cu}(\mathrm{OH})_{2}$. O selênio, presente como selenito $\left(\mathrm{SeO}_{3}{ }^{2-}\right)$, permaneceu em solução [30]. A solução alcalina de selênio foi tratada com sulfito de sódio $\left(\mathrm{Na}_{2} \mathrm{SO}_{3}\right)$, ocorrendo a precipitação de um sólido vermelho:

$$
8 \mathrm{H}_{2} \mathrm{O}+8 \mathrm{SeO}_{3}{ }^{2-}+16 \mathrm{SO}_{3}{ }^{2-} \rightarrow \mathrm{Se}_{8} \downarrow+16 \mathrm{SO}_{4}{ }^{2-}+16 \mathrm{OH}^{-}
$$

O selênio vermelho é uma das formas alotrópicas desse elemento, tendo uma estrutura semelhante àquela do enxofre amarelo $\left(\mathrm{S}_{8}\right)$ [30]. O selênio é um elemento relativamente raro na crosta terrestre, e seus compostos têm alto valo agregado, daí a decisão de recuperá-lo do resíduo. $\mathrm{O}$ resíduo líquido final foi tratado com $\mathrm{H}_{2} \mathrm{SO}_{4} 2$ mol.L $\mathrm{L}^{-1}$ até $\mathrm{pH}$ entre 6 e 8 .

Oxigênio dissolvido na água $(O D)$. Este resíduo foi processado em duas etapas: 
$1^{\text {a }}$ Etapa - remoção do manganês. Esse elemento foi precipitado mediante adição de $\mathrm{NaOH}$ 12,5 mol. $L^{-1}$ até $\mathrm{pH}$ em torno de 10. A adição de solução de peróxido de hidrogênio $\left(\mathrm{H}_{2} \mathrm{O}_{2}\right)$ a $3 \%$ acelerou o escurecimento do precipitado inicial devido à oxidação de $\mathrm{Mn}$ (II) a $\mathrm{Mn}(\mathrm{IV})$ pelo oxigênio atmosférico:

$\mathrm{Mn}^{+2}+2 \mathrm{OH}^{-} \rightarrow \mathrm{Mn}(\mathrm{OH})_{2 \downarrow}$

$\mathrm{Mn}(\mathrm{OH})_{2 \downarrow}+1 / 2 \mathrm{O}_{2} \rightarrow \mathrm{MnO}(\mathrm{OH})_{2 \downarrow}$

O precipitado contendo manganês é filtrado em papel de filtro, e o sobrenadante foi encaminhado para a segunda etapa.

2a etapa - remoção do iodeto. Parte do iodeto pode ser oxidado a iodo pelo $\mathrm{H}_{2} \mathrm{O}_{2}$, caso este seja adicionado. Mesmo não sendo o caso, o oxigênio do ar oxida este íon lentamente, especialmente na presença de luz. A melhor forma de descartar o iodo é na forma do íon iodeto. Então, ao sobrenadante, adicionou-se sulfito de sódio $\left(\mathrm{Na}_{2} \mathrm{SO}_{3}\right)$ sólido, aos poucos, até a desaparição da cor castanha característica do iodo em solução aquosa:

$$
\mathrm{H}_{2} \mathrm{O}+\mathrm{I}_{2}+\mathrm{SO}_{3}{ }^{2-} \rightarrow \mathrm{SO}_{4}{ }^{2-}+2 \mathrm{I}^{-}+2 \mathrm{H}^{+}
$$

$\mathrm{Na}$ sequência, o sobrenadante foi neutralizado com $\mathrm{H}_{2} \mathrm{SO}_{4} 2$ mol.L $\mathrm{L}^{-1}$ até $\mathrm{pH}$ em torno de 7.

Cloreto de zinco. Esse resíduo foi tratado com $\mathrm{NaOH} 12,5$ mol.L $\mathrm{L}^{-1}$, precipitando o metal na forma de hidróxido. $\mathrm{O}$ sobrenadante apresentava $\mathrm{pH}$ em torno de 8; se necessário, ajustou-se o pH com $\mathrm{H}_{2} \mathrm{SO}_{4} 2$ mol.L $\mathrm{L}^{-1}$ para mantê-lo em torno de 7.

$$
\mathrm{ZnCl}_{2}+2 \mathrm{OH}^{-} \rightarrow \mathrm{Zn}(\mathrm{OH})_{2 \downarrow}+2 \mathrm{Cl}^{-}
$$


Deve-se evitar excesso de $\mathrm{NaOH}$ para que o $\mathrm{pH}$, ainda que localmente, fique acima de 13, permitindo a redissolução do $\mathrm{Zn}(\mathrm{OH})_{2}$ como complexo solúvel tetraidroxizincato, $\left[\mathrm{Zn}(\mathrm{OH})_{4}{ }^{2-}\right.$ ] [30].

\subsection{Análise de dos efluentes finais}

Com base na Resolução 357/05 do Conselho Nacional do Meio Ambiente (CONAMA) [31], após os tratamentos apresentados anteriormente, os efluentes foram analisados quanto a quatro requisitos que devem ser satisfeitos simultaneamente para que ele possa ser descartado na pia: a) caráter redox indiferente (placa de toque com $\mathrm{KI}+\mathrm{H}_{2} \mathrm{SO}_{4}$ diluído numa cavidade, e $\mathrm{KMnO}_{4}+\mathrm{H}_{2} \mathrm{SO}_{4}$ diluído numa outra); b) pH entre 5 e 9 (papel indicador universal); ausência de sólidos em suspensão (observação visual de turbidez ou deposição de sólidos após 1 h); d) ausência de cor (inspeção visual). Em seguida, os efleuntes foram analisados por fluorescência de raios-x (equipamento Bruker AXSD5005) para deecção qualitativa dos elementos principais presentes em cada uma das correntes residuais tratadas (o limite de detecção é $0,1 \mathrm{mg} . \mathrm{L}^{-1}$ ). Em caso de detecção, cada elemento identificado será quantificado por meio de uma curva de calibração.

\section{Resultados e discussão}

\subsection{Caracterização visual e físico-química dos efluentes finais dos tratamentos}

Todos os efluentes finais oriundos dos tratamentos aplicados às correntes residuais estão listados na Tabela 3. Exceto no caso do cobre e do manganês, que foram isolados antes de se chegar à etapa final de processamento, a recuperação dos demais elementos sempre deixou como resultado final um efluente tratado. Dentro das condições experimentais deste trabalho, todos esses efluentes atendiam às quatro condições básicas necessárias para descartar um efluente tratado na pia. Para que isso seja possível, é importante ter em mente os seguintes critérios: a) escolha de um agente adequado para isolar o(s) elementos(s) presentes no resíduo. O mais das vezes isso se faz via precipitação de sais ou hidróxidos de produtos de solubilidade muito baixos; b) ajuste do $\mathrm{pH}$ à faixa ideal mediante adição de ácido ou base forte, o que muita vezes serve 
também para precipitar os metais pesados solúveis eventualmente presentes; c) filtração em meio adequado, evitando que parte do precipitado possa passar pelo meio filtrante, o que leva à presença de sólidos em suspensão, turbidez e coloração. As características e a quantidade de preciptiado a ser obtido determinarão a escolha do método de separação sólido-líquido; d) a presença de redutores ou oxidantes no efluente deve ser eliminada de modo a não causar eventuais impactos ambientais no momento do descarte. Para resíduos redutores, $\mathrm{H}_{2} \mathrm{O}_{2}$ e $\mathrm{NaClO}$ (hipoclorito de sódio) são agentes oxidantes adequados pois não geram resíduos de processo. No caso de resíduos oxidantes, sulfito de sódio $\left(\mathrm{Na}_{2} \mathrm{SO}_{3}\right)$, metabissulfito de sódio $\left(\mathrm{Na}_{2} \mathrm{~S}_{2} \mathrm{O}_{5}\right)$, ácido fórmico, ácido oxálico e ácido ascórbico são agentes redutores que não geram resíduos finais de processo. O conhecimento da composição de cada uma das correntes residuais é de fundamental importância para o gerenciamento dos resíduos químicos produzidos, definindo a forma de estocagem e de tratamento dos mesmos.

Além das espécies químicas oriundas de cada resíduo em função da análise que lhe deu origem, outros compostos são adicionados durante o processo de tratamento. No tratamento do resíduo da análise de fosfato, por exemplo, são adicionadas compostos para neutralização $(\mathrm{NaOH})$, precipitação das espécies $\left(\mathrm{BaCl}_{2}\right)$ e posterior eliminação de excesso dos elementos adicionados em solução $\left(\mathrm{Na}_{2} \mathrm{SO}_{4}\right)$. Deve-se tomar cuidado para que nehuma das espécies químicas reconhecidamente poluidoras sejam descartadas em uma ou várias das etapas do tratamento de um resíduo químico. De um modo geral, não se recomenda a adição de metal pesado para tratamento de resíduos, exceto se ele é oriundo de uma outra corrente residual (quando os resíduos se tratam mutuamente).

A fluorescência de raios-x não acusou nenhum dos elementos de interesse em concntração detectáveis $(<0,1 \mathrm{mg} / \mathrm{L}$ ), salvo no caso do iodo (que é descartado junto com o efluente tratado) e do etanol (substância líquida). Esse resultado enquadra os efluentes para descarte segundo a Resolução 357/05 do CONAMA [31]. 
Tabela 3: Caracterização dos produtos finais após os tratamentos

\begin{tabular}{ccc}
\hline $\begin{array}{c}\text { Elemento ou } \\
\text { composto de } \\
\text { interesse }\end{array}$ & $\begin{array}{c}\text { Aspecto visual do } \\
\text { efluente tratado }\end{array}$ & $\begin{array}{c}\text { Aspecto do produto contendo o elemento ou } \\
\text { composto de interesse }\end{array}$ \\
\hline $\mathrm{Mo}$ & Solução incolor & Não foi isolado o produto $\left(\mathrm{BaMoO}_{4}\right)$ \\
$\mathrm{Sb}$ & Solução incolor & Não foi isolado o produto $(\mathrm{SbOOH}+\mathrm{SbOCl})$ \\
$\mathrm{Hg}$ & Solução incolor & Preciptiado branco $\left(\mathrm{Hg}(\mathrm{OH})_{2}\right)$ \\
$\mathrm{Etanol}$ & Solução verde & Liquido transparente e incolor \\
$\mathrm{Zn}$ & Solução incolor & Preciptiado branco $\left(\mathrm{Zn}(\mathrm{OH})_{2}\right)$ \\
$\mathrm{Cu}$ & Não houve & Preciptiado azul $\left(\mathrm{Cu}(\mathrm{OH})_{2}\right)$ \\
$\mathrm{Se}$ & Solução incolor & Preciptiado vermelho $\left.(\mathrm{Se})_{8}\right)$ \\
$\mathrm{Mn}$ & Não houve & Preciptiado marrom $\left(\mathrm{MnO}(\mathrm{OH})_{2}\right)$ \\
$\mathrm{I}$ & Solução incolor & Não foi isolado o produto $\left(\mathrm{I}^{-}\right)$ \\
\hline & & \\
\hline
\end{tabular}

\subsection{Volume final dos efluentes tratados}

Um dos aspectos mais importantes no tratamento de quaisquer resíduos químicos é o efeito de diluição durante as várias etapas do tratamento. Em geral, o volume do efluente final é maior do que o do resíduo inicial (15-20\% em nosso caso). Por isso, sempre que possível, devese utilizar soluções mais concentradas do agente de tratamento. Por exemplo, no caso da neutralização, pode-se utilizar $\mathrm{NaOH}, \mathrm{Na}_{2} \mathrm{CO}_{3}$ ou $\mathrm{NaHCO}_{3}$ sólidos, exigindo apenas uma agitação efetiva para homogeneização e controlar o efeito da formação de espuma. Para soluções alcalinas, podem-se empregar ácidos de maior concentração apesar da manipulação ser mais 
delicada. Na neutralização, deve-se seguir esta regra: a solução mais concentrada é adicionada paulatinamente e sob agitação à solução mais diluída.

A única exceção a essa tendência foi o resíduo de etanol; a recuperação desse solvente levou à redução do volume de resíduo final. Esse comportamento ocorre quando da recuperação de solventes ou da evaporção de soluções para cristalização de algum soluto dissolvido [2,3,6-9].

\subsection{Destino final dos produtos sólidos e líquidos isolados}

O etanol recuperado foi rotulado como solvente de lavagem e limpeza. Os precipitados de hidróxido de zinco (ou de cobre) foram utilizados na síntese de cloretos desses metais (mediante adição da quantidade adequada de $\mathrm{HCl}$ para dissolução); eles foram encaminhados a laboratórios de ensino de química analítica qualitativa e química geral. O selênio foi encaminhado a grupos de pesquisa que sintetizaram o $\mathrm{SeO}_{2}$ (reagente útil em sínteses orgânicas). Os resíduos contendo $\mathrm{Mo}, \mathrm{Sb}$ e $\mathrm{Mn}$ podem tanto ser enviados à incineração (as cinzas são destinadas a aterros industriais Classe I) [32], como a coprocessamento em fornos de cimenteiras (material mineralizante), segundo a Resolução 264/99 do CONAMA [33]. O resíduo contendo mercúrio somente pode ser enviado diretamente a aterro industrial para resíduos Classe I, devendo ainda ser encapsulado antes dessa destinação final [32].

O laboratório de Limnologia da UFRJ encaminha o material identificado de acordo com as recomendações da Comissão de Biossegurança do Centro de Ciências da Saúde (CCS) da Universidade, que realiza duas vezes ao ano uma coleta dos resíduos químicos dos laboratórios deste centro, de acordo com a seguinte classificação: a) resíduos inorgânicos sólidos ou pastosos; b) resíduos orgânicos sólidos ou líquidos (isentos de compostos halogenados e/ou fosforados); c) resíduos orgânicos sólidos ou líquidos halogenados e/ou fosforados; d) resíduos não identificados.

\subsection{O comportamento do fator humano no processo de gestão}


Com a progressiva implementação do programa de gerenciamento de resíduos químicos no Laboratório de Limnologia, observou-se uma mudança de postura pelos seus membros, que abandonaram o procedimento de descarte puro e simples na pia ou no lixo comum, cultura estabelecida quando eles eram alunos de disciplinas experimentais de cursos de nível técnico e mesmo superior. A realização de seminários e reuniões internas, o mapeamento das fontes geradoras, o estabelecimento de um sistema de identificação de cada corrente, e o estabelecimento de roteiros de tratamento testados, reavaliados e aprimorados foram fundamentais para o envolvimento de todos com esta atividade. Os resultados iniciais do programa de gestão sempre foram colocados de forma clara para todos, valorizando o esforço individual e coletivo, identificando casos de estratégias bem sucedidas e outros em que ainda são necessários estudos adicionais.

$\mathrm{Na}$ área da saúde a gestão dos resíduos químicos é parte integrante de um universo mais amplo, que é a gestão dos resíduos de saúde (RSS). De acordo com a Resolução RDC 306/04 da Agência Nacional de Vigilância Sanitária (ANVISA) [34] e a Resolução 358/05 do CONAMA [35], os RSS são compostos por cinco tipos de resíduos, sendo que os químicos integram o grupo B. Portanto, uma boa gestão dos RSS exige a implementação de um programa que contemple os resíduos químicos. Da mesma forma, a gestão desses resíduos minimiza possíveis impactos ambientais, dentro das diretrizes da Norma ABNT ISO 14001 [17,36]. Esses fatos foram colocados de forma bem objetiva, incentivando a todos a manter e a aprimorar as rotas de tratamento desenvolvidas.

Uma vez cumprida essa etapa busca-se agora minimizar a geração dos resíduos, tendo como foco a redução da toxicidade e/ou do volume de geração. Uma menor geração de resíduos reduz o custo de tratamento do mesmo, independente da destinação final a ser dada a ele. Para isso, uma possibilidade é reduzir a escala de trabalho em um dado procedimento, o que nem sempre é possível. Outra possibilidade, dentro da filosofia da chamada "produção mais limpa" $(\mathrm{P}+\mathrm{L})$, é a substituição de metodologias de análise por outras que usem reagentes menos tóxicos e/ou menores quantidades de amostra. Este campo demanda pesquisa e desenvolvimento para se chegar a um resultado concreto. O Laboratório de Limnologia disponibilizou seus resíduos na bolsa de resíduos da Federação das Indústrias do Estado do Rio de Janeiro (FIRJAN) [37]. De acordo com a própria FIRJAN, trata-se de uma iniciativa que busca promover a livre negociação 
entre indústrias, conciliando ganhos econômicos com ganhos ambientais, a partir da troca de informações sobre os resíduos disponíveis. Esta é uma prática corrente nos Estados Unidos, Europa e Japão.

\section{Conclusões}

Diferente de muitas experiências sobre tratamento de resíduos químicos que trabalham com material de aulas práticas ou de rotinas de laboratório de pesquisa em química, este trabalho apresenta um caráter diferenciado na medida em que a diversidade de amostras analisadas pelos métodos empregados no Laboratório de Limnologia gera também uma diversidade de resíduos dentro da mesma corrente. Isso exige a adoção de procedimentos que possibilitem uma padronização dos tratamentos em função das análises que originaram tais resíduos, independente de sua origem. Para alcançarmos esta condição, foi fundamental a adoção de uma postura participativa por parte de todos os membros do laboratório, em que todos assimilassem os princípios fundamentais da gestão adequada dos resíduos que eles mesmos produziam. Sendo assim, o princípio da corresponsabilidade, no qual o gerador do resíduo, em vários níveis de escala de participação, deve participar e acompanhar todo o processo de tratamento e/ou destinação final do resíduo químico produzido, leva à necessidade de que toda a hierarquia do laboratório (coordenadores, docentes, técnicos e alunos) se envolva neste sistema. Como os RSS englobam cinco tipos distintos, a experiência com os resíduos químicos pode servir para melhorar ou mesmo implementar sistemas semelhantes de gestão para os demais tipos de resíduos.

\section{Agradecimentos}

Os autores agradecem a toda equipe do laboratório de Limnologia, pela dedicação e colaboração na implementação do sistema de gerenciamento de resíduos químicos. A Finep, FAPERJ, CNPq e Petrobras, através dos recursos disponibilizados para realização destas atividades. 


\section{Referências}

[1] A. F. Silva, T. R. S. Soares; J. C. Afonso; Química Nova na Escola, (32) (2010) 37.

[2] L. B. A. Alberguini, L. C. Silva, M. O. O. Rezende, Quim. Nova, (26) (2003) 291.

[3] W. F. Jardim, Quim. Nova, (21) (1998) 671.

[4] B. Ray, J. Chem. Educ, (80) (2003) 45.

[5] The Committee of Chemical Safety, 2010 Spring National Meeting, American Chemical Society: Washington, 2010, 66 p.

[6] L. C. F. Pimentel, C. R. Chaves, L. A. A. Freire, J. C. Afonso, Quim. Nova, (29) (2006) 1138.

[7] J. C. Afonso, L. A. Noronha, R. P. Felipe, N. Freidinger, Quim. Nova, (26) (2003) 602.

[8] J. C. Afonso, J. A. Silveira, A. S. Oliveira, R. M. G. Lima, Quim. Nova, (28) (2005) 65.

[9] J. C. Afonso, D. F. Araújo, Rev. Quim. Ind., (723) (2005) 12.

[10] A. E Gerbase, J. R. Gregório, T. Calvete, Quim. Nova, (29) (2006) 397.

[11] D. Imbroisi, A. J. M. Guaritá-Santos, S. S. Barbosa, S. F. Shintaku, H. J. Monteiro, G. A. E. Ponce, J. G. Furtado, C. J. Tinoco, D. C. Mello, P. F. L. Machado, Quim. Nova, (29) (2006) 404.

[12] A. S. Demaman, S. Funk, L. V. Hepp, A. M. S. Adário, S. B. C. Pergher, Quim. Nova, (27) (2004) 674.

[13] J. A. Bendassolli, G. A. Tavares, R. F. Ignoto, A. L. R. M. Rosseti, Quim. Nova, (26) (2003) 578.

[14] Z. T. C. Leite, S. Alcantara, J. C. Afonso, Quim. Nova, (31) (2008) 1892.

[15] R. Felisberto, L. O. Vieira, A. Couto, R. Schuh, C. T. Albino, D. B. Libardi, A. C. B. Cunha, Quim. Nova, (31) (2008) 174.

[16] P. C. Giloni-Lima, J. A. Lima, Quim. Nova, (31) (2008) 1595. 
[17] E. S. Gil, C. F. D. Garrote, E. C. Conceição, M. F. Santiago, A. R. Souza, Rev. Bras. Cienc. Farmacêuticas, (43) (2007) 19.

[18] D. L. Adams; J. Chem. Educ., (76) (1999) 1088.

[19] M. A. Armour, L. M. Browne, G. L. Weir, J. Chem. Educ., (62) (1985) A93.

[20] J. A. Bendassolli, E. Maximo, G. A. Tavares, R. F. Ignoto, Quím. Nova, (26) (2003) 612.

[21] V. F. Farjalla, B. M. Faria, F. A. Esteves, R. L. Bozelli, Oecologia Brasiliensis, (9) (2001) 65.

[22] Associação Brasileira de Normas Técnicas, NBR 10004, ABNT, Rio de Janeiro, 2004.

[23] Associação Brasileira de Normas Técnicas, NBR 12235, ABNT, Rio de Janeiro, 1992.

[24] Norma Regulamentadora $\mathrm{N}^{0} 15$ do Ministério do Trabalho e Emprego, Brasil. (http://www.mte.gov.br/legislacao/normas_regulamentadoras/nr_15.pdf, acessado em junho 2010).

[25] H. L. Golterman, R. S. Clymo, M. A. M. Ohnstad, Methods of physical and chemical analysis of freshwaters, Blackwell: Oxford, 1978.

[26] F. J. H. Mackereth, J. Heron, J. F. Talling, Water analysis: some revised methods for limnologists, Freshwater Biological Association, scientific publication $\mathrm{n}^{\mathrm{o}} 36$, Cumbria and Dorset, 1978.

[27] Protocol Handbook for Nitrogen Cycling In Estuaries (NICE), Ed. National Environmental Research Institute, Denmark - Department of Lake and Estuarine Ecology, 2000.

[28] H. W. Fassbender, Simulate P-Bestinmung in N-Kjeldahl Ausfschlub von Bodenproben. Die Phosphörsäure; Verlag: Berlin, 1973.

[29] S. E. Allen, H. M. Grimshaw, J. A. Parkinson, C. Quarmby, Chemical analysis of ecological material, Blackwell Scientific Publications: Oxford, 1974.

[30] J. E. Brady, F. Senese, Química, a Matéria e suas Transformações, tradução da 5a edição norte-americana; Livros Técnicos e Científicos: Rio de Janeiro, 2009, cap. 21. 
[31] Resolução 357/05 do Conselho Nacional do Meio Ambiente, 17/03/2005, Diário Oficial da União, 18/03/2005.

[32] D. V. Figueredo, Manual para Gestão de Resíduos Químicos Perigosos de Instituições de Ensino e Pesquisa, Conselho Regional de Química de Minas Gerais: Belo Horizonte, 2006.

[33] Resolução 264/99 do Conselho Nacional do Meio Ambiente, 26/08/1999, Diário Oficial da União, 20/03/2000.

[34] Resolução RDC 306/04 da Agência Nacional de Vigilância Sanitária, 07/12/2004, Diário Oficial da União, 10/12/2004.

[35] Resolução 358/05 do Conselho Nacional do Meio Ambiente, 29/04/2005, Diário Oficial da União, 04/05/2005.

[36] Associação Brasileira de Normas Técnicas, NBR ISO 14001, ABNT, Rio de Janeiro, 2004.

[37] http://www.firjan.org.br/notas/cgi/cgilua.exe, acessada em maio de 2010. 